Monika Palowski, Johanna Gold \& Gabriele Klewin

\title{
Gemeinsame Praxisforschung statt Be-Forschung: Die Bielefelder Versuchsschulen und ihre Wissenschaftlichen Einrichtungen
}

\section{Zusammenfassung}

Der Beitrag skizziert die Konzeption und Organisation von Forschungs- und Entwicklungsprozessen an den beiden Bielefelder Versuchsschulen Laborschule und Oberstufen-Kolleg und ihren Wissenschaftlichen Einrichtungen. Dazu werden nach einer kurzen historischen Einordnung die Rahmenbedingungen, in denen die Versuchsschulen und ihre Forschungseinrichtungen gemeinsam Praxisforschung betreiben, vorgestellt und durch zwei Beispiele für Forschungs- und Entwicklungsprojekte verdeutlicht.

Schlüsselwörter: Versuchsschulen, Wissenschaftliche Einrichtungen, Universität, Kooperation, Praxisforschung

\section{Collaborative Action Research instead of External Inquiry: The Bielefeld Experimental Schools and Their Research Departments}

\section{Summary}

This article outlines the concept and organization of joint research and development at the two experimental schools (Laborschule and Oberstufen-Kolleg) in Bielefeld. Both schools have research departments belonging to Bielefeld University. Teachers from the schools and researchers from University cooperate in joint research and development projects. Following a brief historical overview, we describe the conditions underlying this concept of permanent cooperation and illustrate them, using two examples of research projects.

Keywords: experimental schools, research departments, university, cooperation, teacher research 


\section{Einführung}

Die beiden Bielefelder Versuchsschulen Laborschule (Primarstufe und Sekundarstufe I) und Oberstufen-Kolleg (Sekundarstufe II) stellen eine Besonderheit in der deutschen Schullandschaft dar: Beide verfügen über Wissenschaftliche Einrichtungen und damit über dauerhaft institutionalisierte Kooperationen mit einer Universität. Gemeinsam betreiben die Schulen und die Wissenschaftlichen Einrichtungen schon seit über 40 Jahren Praxisforschung mit unterschiedlichen Akzenten und blicken somit auf langjährige Erfahrungen in diesem Feld zurück. Der Beitrag fokussiert mit Blick auf den Themenschwerpunkt dieses Heftes die Besonderheiten hinsichtlich der Forschung und vernachlässigt die Besonderheiten der pädagogischen Programme (siehe hierzu Hahn \& Oelkers, 2012, bzw. Thurn \& Tillmann, 2011). Er skizziert kurz die historische Entwicklung der Schulen und ihrer Wissenschaftlichen Einrichtungen, um daraufhin die aktuelle Konzeption von Forschung und Entwicklung zu schildern und anhand von zwei Beispielen zu illustrieren.

\section{Wer, wie, was und wann}

Die Bielefelder Versuchsschulen wurden 1974 von Hartmut von Hentig als zentrale Wissenschaftliche Einrichtungen der Universität Bielefeld gegründet und waren somit ursprünglich Teil der Universität. Alle Lehrkräfte waren zugleich Forscherinnen, die ihre Praxis projektförmig und mit einem hohen Anteil ihres Deputats erforschten; insofern waren beide Schulen von Beginn an explizit an einem Forschungsschwerpunkt mit hohem Anwendungsbezug orientiert.

In der Laborschule erfolgte im Zuge einer umfassenden Umstrukturierung, die bereits 1986 mit ersten Vereinbarungen zwischen der Laborschule und der Fakultät für Pädagogik ${ }^{1}$ begann und schließlich 1992 in einem Grundlagenerlass festgehalten wurde, eine Trennung zwischen der Versuchsschule und der Wissenschaftlichen Einrichtung Laborschule ${ }^{2}$ (Tillmann, 2011). Im Oberstufen-Kolleg fand diese Aufteilung erst 2007 statt (Keuffer \& Klewin, 2009). Die Schulen wurden dem Zuständigkeitsbereich des Ministeriums für Schule und Weiterbildung des Landes Nordrhein-Westfalen ${ }^{3}$ unterstellt, während die Wissenschaftlichen Einrichtungen an der Universität angesiedelt blieben. Sie sind inzwischen Wissenschaftliche Einrichtungen der Fakultät für Erziehungswissenschaft an der Universität Bielefeld und sind durch je eine Professur und mehrere wissenschaftliche Mittelbau-Stellen dort verankert.

12008 erfolgte an der Universität Bielefeld eine Umbenennung der damaligen Fakultät für Pädagogik in Fakultät für Erziehungswissenschaft.

2 Soweit nicht anders spezifiziert, sind „Laborschule“ und „Oberstufen-Kolleg“ im Folgenden jeweils als Überbegriffe für die jeweilige Versuchsschule und die ihr zugehörige Wissenschaftliche Einrichtung zu verstehen.

3 Das jetzige Ministerium für Schule und Bildung des Landes Nordrhein-Westfalen. 
Die Wissenschaftliche Leiterin bzw. der Wissenschaftliche Leiter der Laborschule wird für jeweils eine Amtszeit von fünf Jahren vom Vorstand der Wissenschaftlichen Einrichtung gewählt (Tillmann, 2011); im Oberstufen-Kolleg hingegen ist das Amt der Wissenschaftlichen Leiterin bzw. des Wissenschaftlichen Leiters dauerhaft mit einer Professur verbunden. ${ }^{4}$

Die Lehrkräfte der Versuchsschulen und die Mitarbeiter*innen der Wissenschaftlichen Einrichtungen kooperieren in Forschungs- und Entwicklungsprojekten und im Rahmen der Schulentwicklung. Insofern lässt sich heute von vier Institutionen sprechen, nämlich den beiden Versuchsschulen und ihren Wissenschaftlichen Einrichtungen, die jedoch jeweils eng zusammenarbeiten. Die Aufteilung bei gleichzeitiger gemeinsamer Forschungs- und Entwicklungsarbeit brachte neue Herausforderungen mit sich, die insbesondere in den unterschiedlichen Logiken des Wissenschafts- und des Schulsystems begründet liegen. Gleichzeitig erforderte die Aufteilung neue, gemeinsame Gremien sowie veränderte Verfahren der Beantragung, Bewilligung, Durchführung und Berichtslegung der Forschungs- und Entwicklungsprojekte (S. u.), wobei gerade letztere kontinuierlich weiterentwickelt werden.

Auch wenn, wie oben erwähnt, die besonderen pädagogischen Programme der Schulen hier nicht dargestellt werden können, soll jedoch ihre Rolle in der Lehrerausbildung kurz erwähnt werden. Das Oberstufen-Kolleg bildet Praxissemesterstudierende sowie Referendar*innen analog zur Ausbildung an Regelschulen aus; an der Laborschule werden nur Praxissemesterstudierende und bisher keine Referendar*innen ausgebildet. Die Ergebnisse der Forschungs- und Entwicklungsprojekte finden Eingang in die Lehrer*innen(aus)bildung durch die universitäre Lehre der Wissenschaftlichen Mitarbeiter*innen und auch immer wieder durch Lehrkräfte der Schule sowie durch Publikationen in wissenschaftlichen und schulpraktischen Diskursen. Darüber hinaus werden aus verschiedenen Forschungs- und Entwicklungsprojekten heraus Fortbildungsangebote für die dritte Phase durchgeführt. Dies geschieht derzeit verstärkt in der Kooperation mit dem Projekt Biprofessional im Rahmen der gemeinsamen Qualitätsoffensive Lehrerbildung von Bund und Ländern (FKZ 01JA1608).

\section{Modell der Forschung und Entwicklung}

Das in den 1970er-Jahren ursprünglich als „Lehrer-Forscher-Modell“ entstandene Konzept von Forschung und Entwicklung in den Versuchsschulen Laborschule und Oberstufen-Kolleg hat über die Jahre verschiedentlichen Wandel erfahren und in beiden Schulen mit ihren jeweiligen Wissenschaftlichen Einrichtungen eine leicht un-

4 Trotz genereller Übereinstimmungen zwischen Laborschule und Oberstufen-Kolleg, sowohl in den Strukturen als auch in der Forschungsausrichtung, sind auf der Detailebene einige Unterschiede zu finden. 
terschiedlich akzentuierte Entwicklung genommen (Jung-Paarmann, 2014a, 2014b; Huber, 2004). Im Wesentlichen stimmt das Vorgehen jedoch überein (Klewin, Schumacher \& Textor, 2016). So orientieren sich die aktuellen Forschungs- und Entwicklungsmodelle an der Aktions- bzw. Praxisforschung ${ }^{5}$ (Altrichter, Posch \& Spann, 2018), was sich z. T. auch in der Verwendung des Begriffs „Praxisforschung“ statt „Lehrer*innenforschung“ zeigt. Kennzeichnend dafür ist die Forschung durch die Lehrkräfte selbst, an Gegenständen oder Problemstellungen, die für ihren Schulalltag eine besondere Relevanz haben. Die Ergebnisse sollen in der Folge zur Weiterentwicklung von Schule und Unterricht sowohl im eigenen Haus als auch im Regelschulsystem genutzt werden (z.B. Meyer, 2010) und Eingang in den wissenschaftlichen Diskurs finden. Die Unterschiede zur bei Altrichter et al. (2018) beschriebenen Aktionsforschung liegen

- in der dauerhaften und institutionalisierten Kooperation der Lehrkräfte mit Wissenschaftler ${ }^{\star}$ innen einer eigenen Einrichtung für die Forschung in den Schulen, über die auch eine dauerhafte Kooperation mit der Fakultät für Erziehungswissenschaft gesichert ist;

- in der Kontinuität der Forschung, die u. a. mehrjährige Forschungszyklen und die Möglichkeit, Folgeanträge zu stellen, ermöglicht;

- in festgeschriebenen Verfahren zur Beantragung, Beratung und Bewilligung von Projekten und in der Berichtspflicht (Gemeinsame Leitung des OberstufenKollegs Bielefeld, 2017; Tillmann, 2011), worin sich ein wesentliches Merkmal der Qualitätssicherung findet;

- in der Sicherstellung einer Anbindung an relevante akademische, administrative und politische Diskurse durch Beiräte und Wissenschaftliche Mitarbeiter*innen;

- in den zur Verfügung stehenden Ressourcen für Lehrkräfte, die sich an Forschung und Entwicklung beteiligen (Grundordnung des Oberstufen-Kollegs: MSW NRW, 2007); sowie

- im gemeinsamen Versuchsauftrag, der Zielsetzung und Rahmen für die Forschung und Entwicklung absteckt und für die Versuchsschulen und ihre Wissenschaftlichen Einrichtungen jeweils identisch formuliert ist (Präambeln der Grundordnung des Oberstufen-Kollegs und Verwaltungs- und Benutzungsordnung der Wissenschaftlichen Einrichtung Oberstufen-Kolleg: MSW NRW, 2007; Grundlagenerlass für die Aufgaben und die Zusammenarbeit von Versuchsschule, Wissenschaftlicher Einrichtung, Gemeinsamer Leitung und Wissenschaftlichem Beirat: Laborschule Bielefeld, 2012).

5 Altrichter und Feindt folgend betrachten wir Praxisforschung, Lehrer*innenforschung, Aktionsforschung, Handlungsforschung, teacher research oder practitioner research als eine „,Familie von Konzepten, die eine gemeinsame Zielrichtung haben“" (Altrichter \& Feindt, 2004, S. 84). 
Wie oben bereits beschrieben, machte die Trennung von schulischem und wissenschaftlichem Teil die Einrichtung neuer Gremien erforderlich, die die gemeinsame (Forschungs-)Arbeit steuern. Das jeweils zentrale Gremium ist sowohl an der Laborschule als auch am Oberstufen-Kolleg die jeweilige Gemeinsame Leitung, in der Versuchsschule und Wissenschaftliche Einrichtung zusammenarbeiten. Sie trifft Entscheidungen über Forschungs- und Entwicklungsfragen und ebenfalls über die dafür zur Verfügung stehenden Ressourcen.

Über die Wissenschaftlichen Mitarbeiter*innen der beiden Wissenschaftlichen Einrichtungen hinaus sichern die jeweiligen Vorstände der Wissenschaftlichen Einrichtungen die Anbindung an die und die Vernetzung mit der Fakultät für Erziehungswissenschaft, da die Vorstandsmitglieder in der Regel der Fakultät angehören, jedoch keine Angehörigen der Einrichtungen sind.

Die Organisation der Antragsphase für Forschungs- und Entwicklungsprojekte und der gemeinsamen Arbeit in diesen Projekten liegt bei den Wissenschaftlichen Einrichtungen. Nach gemeinsamer Antragsstellung von Lehrkräften und Wissenschaftlichen Mitarbeiter*innen erfolgen die Beratung in den Beiräten sowie die Entscheidung über Annahme oder Ablehnung des Antrags durch die Gemeinsamen Leitungen. Die Schulleitungen beauftragen schließlich in Absprache mit der Wissenschaftlichen Leitung die Lehrkräfte mit der Arbeit in den Projekten. Sowohl in der Laborschule als auch im Oberstufen-Kolleg stehen für die Lehrkräfte Ressourcen für die Forschungs- und Entwicklungsarbeit zur Verfügung. In der Regel beträgt der Umfang zwischen einer und drei Deputatsstunden.

Die Qualitätssicherung während eines Forschungszyklus geschieht sowohl durch die Wissenschaftlichen Einrichtungen als auch durch die Wissenschaftlichen Beiräte, in denen Zwischen- und Abschlussberichte bzw. die Produkte der Projekte, wie Publikationen, Unterrichtsmaterialien, Fortbildungen u.a., beraten werden und die die Anbindung an relevante Diskurse der Bildungswissenschaft, -administration und -politik sicherstellen. In den einzelnen Projekten sind häufig die Wissenschaftlerinnen dafür zuständig, in methodischen Fragen und bei wissenschaftlichen Publikationen zu beraten und zu unterstützen; viele andere Aufgaben, wie z. B. die Entwicklung von Fragestellungen und Designs sowie die Durchführung von Erhebungen, werden - je nach Projekt - kooperativ oder vollständig von den Lehrkräften durchgeführt; hier existieren unterschiedliche Varianten (vgl. Hahn, Klewin, Koch, Kuhnen, Palowski \& Stiller, im Druck). 


\section{Beispiel eines Forschungs- und Entwicklungsprojektes am Oberstufen-Kolleg: „Innere Differenzierung“}

Als Beispiel für die Forschungsarbeit am Oberstufen-Kolleg und ihre Verknüpfung mit Prozessen der Schulentwicklung eignet sich das Projekt „Innere Differenzierung in der Sekundarstufe II“ (vgl. Boller \& Lau, 2010). Dieses Projekt formierte sich 2006 mit der expliziten Zielsetzung, die Arbeit am damaligen Schwerpunkt der Schulentwicklung, dem produktiven Umgang mit Heterogenität, durch Recherchen und Erhebungen innerhalb und außerhalb des Hauses vorzubereiten, Lehrkräfte des Oberstufen-Kollegs zur Erprobung neuer Methoden der inneren Differenzierung anzuregen und auf dieser Grundlage Fortbildungskonzepte $\mathrm{zu}$ entwickeln und durchzuführen. Das Projekt war dabei teils konstant, teils wechselnd von Lehrkräften der Versuchsschule und Mitarbeiter*innen der Wissenschaftlichen Einrichtung besetzt und arbeitete über drei jeweils zweijährige Forschungszyklen hinweg.

Vor allem im ersten Zyklus (2006-2008) kooperierte das Projekt eng mit der Koordinierungsgruppe Schulentwicklung, denn hier lag der Fokus auf einer „Professionalisierung der Lehrenden [...] im Umgang mit heterogenen Lerngruppen und einer Weiterentwicklung der Möglichkeiten zur individuellen Förderung aller Lernenden“ (Bathe, Bernard, Boller, Kemper, Lau \& Wäcken, 2010, S. 2). Im darauffolgenden Forschungszyklus (2008-2010) wurde mit „Formen der Inneren Differenzierung und [der] Gestaltung von Lernarrangements für eine heterogene Schülerschaft in der Versuchsschule Oberstufen-Kolleg sowie in der Sekundarstufe II des Regelsystems“ (ebd., S. 5) die Reichweite der Arbeit auf den externen Transfer ausgedehnt; in diesem Zyklus entstand u.a. das Praxishandbuch Innere Differenzierung in der Sekundarstufe II (Boller \& Lau, 2010), das sich mit bildungstheoretischer Rahmung und methodischen Anregungen aus dem Oberstufen-Kolleg an alle Lehrkräfte der Oberstufe richtet. Im dritten und letzten Projektzyklus (2010-2012) standen externe Fortbildungen und Workshops im Fokus, die jeweils mit Hilfe einer empirischen Bedarfsanalyse vorbereitet und nachfragespezifisch angepasst sowie in der Mehrzahl der Fälle durch eine umfangreiche Evaluation nachbereitet wurden (vgl. Lau \& Große-Klußmann, 2012).

Das hier nur kurz skizzierte Projekt illustriert eine der verschiedenen am OberstufenKolleg vorfindbaren Varianten des Praxisforschungszyklus (vgl. Klewin \& Palowski, 2018): Ausgehend von dem innerschulischen Bedarf nach forschender Vorbereitung eines Schulentwicklungsprozesses erarbeiteten Lehrkräfte und Wissenschaftler*innen gemeinsam ein Konzept für diesen Prozess; im Anschluss an diese sehr stark hausintern orientierte Entwicklungsphase erfolgte dann eine extern orientierte Disseminationsphase, wobei durchgehend die Praxisforschung - zunächst zur Ermittlung von Forschungsstand und bereits vorhandenem Methodenrepertoire im Haus, dann zur Bedarfsermittlung und Evaluation von Fortbildungen außerhalb des Hauses - der 
primäre Bearbeitungsmodus, gleichzeitig jedoch eine enge Verflechtung mit hausinterner Schulentwicklung kennzeichnend war. Besonderes Merkmal dieses Projektes ist zudem der breite Transfer in die externe schulische Praxis.

\section{Verbindung zweier Forschungs- und Entwicklungsprojekte an der Laborschule: „Absolvent ${ }^{*}$ innenstudie“ und „Übergang der Laborschüler ${ }^{\star}$ innen von der Sekundarstufe $I$ in weitere schulische und berufliche Lebensumwelten“"}

Die „Absolvent*innenstudie“ ist ein seit 1985 fortlaufendes Forschungs- und Entwicklungsprojekt der Laborschule. In ihrem Rahmen werden Schüler*innen am Ende ihrer Schullaufbahn an der Laborschule und nach drei Jahren in einer neuen Lernumgebung um eine Einschätzung bezüglich konstituierender Merkmale der Laborschulpädagogik und erworbener Kompetenzen gebeten. Darüber hinaus werden persönliche Merkmale der Schüler*innen wie Selbstwertgefühl, Leistungsverständnis, Selbstkonzept und Interesse sowie Angaben zum familiären Hintergrund erhoben. Es handelt sich um eine quantitative Längsschnittstudie, deren Daten mit Hilfe standardisierter Fragebögen generiert werden. Der Datenpool wächst durch die turnusmäßigen Erhebungen kontinuierlich an. Zurzeit umfasst der Datensatz des ersten Befragungszeitpunktes 1.596 Fälle, der des zweiten Befragungszeitpunktes 939. Die „Absolvent*innenstudie“ generiert zum einen Hinweise zur Entwicklung schulischer Praxis und zum anderen zu weiterführender Forschung. Das aktuelle, an die Befunde der „Absolvent*innenstudie“ anknüpfende Projekt „Übergang der Laborschülerinnen von der Sekundarstufe I in weitere schulische und berufliche Lebensumwelten“ beleuchtet die Wahrnehmung und retrospektive Bewertung der Übergangsphase von der Laborschule in weitere schulische und berufliche Lebensumwelten von Seiten der Schüler*innen. Diese Übergangsphase als Herausforderung für die Schulentwicklung zu begreifen und zu bearbeiten, ist die Zielsetzung dieses Projektes. Die Schüler*innen der Laborschule verlassen nach der 10. Jahrgangsstufe - nach insgesamt 11 Jahren - die Schule und machen sich auf den Weg in andere schulische oder berufliche Lebensumwelten. Diese „Statuspassage“ (Bosse \& Kempf, 2013) stellt für sie ein bedeutsames Lebensereignis dar, und ihre Entscheidungen in dieser Phase haben wesentlichen Einfluss auf ihren weiteren Bildungs- und Lebensweg. Für Schule stellt somit die Vorbereitung auf die und die Unterstützung während der Übergangsphase einen substanziellen Teil des Bildungsauftrags dar.

Im ersten Forschungszyklus (2015-2018) ${ }^{6}$ wurden das Untersuchungsdesign (Gruppendiskussionen mit Absolvent*innen in zwei Kohorten zu zwei Zeitpunkten) entwickelt und die Datenerhebung zum ersten Messzeitpunkt für beide Kohorten

6 Ein Forschungszyklus in der Laborschule umfasst im Normalfall zwei Jahre; der Zyklus 2015-2018 wurde um ein Jahr verlängert. 
und zum zweiten Messzeitpunkt für die erste Kohorte durchgeführt. Beim zweiten Messzeitpunkt wurden im Sinne der Stimulated-Recall-Methode (Gass \& Mackey, 2000) Interviewpassagen aus dem ersten Messzeitpunkt als Erzählstimuli aufbereitet und verwendet. Zum zweiten Messzeitpunkt wurden die Befragten zusätzlich gebeten, konkrete Vorschläge für die pädagogische Entwicklung und Ausgestaltung der Übergangphase zu machen, und so aktiv in den Schulentwicklungsprozess einbezogen. Der aktuelle Forschungszeitraum (2018-2020) dient zum Abschluss der Datenerhebung, zu weiterer Datenanalyse und zur Aufarbeitung der Ergebnisse. Das Projekt wird mit einer Publikation in Form eines Sammelbandes abgeschlossen.

\section{Resümee}

Die beiden beispielhaft skizzierten Forschungs- und Entwicklungsprojekte zeigen das Potenzial von Praxisforschung an den Bielefelder Versuchsschulen. Praxisforschung ist bzw. kann eng mit Schulentwicklung verknüpft sein und Ergebnisse, Konzepte und Materialien hervorbringen, die in Schulentwicklungsprozessen genutzt werden können. Zugleich entstehen aus vielen Projekten auch Produkte, die in Regelschulen transferiert werden und somit die dortige Schulentwicklung befördern können. Beides wird, und dies zeigen die Beispiele besonders deutlich, durch die Kontinuität der gemeinsamen Forschungs- und Entwicklungsarbeit von Schulen und Wissenschaftlichen Einrichtungen in mehrjährigen Forschungszyklen ermöglicht, aus der vertiefte Erkenntnisse resultieren und die die Rahmenbedingungen für internen und externen Transfer bietet.

Mit Laborschule und Oberstufen-Kolleg wurden in diesem Beitrag zwei Versuchsschulen beschrieben, die sowohl hinsichtlich der Forschungsausrichtung als auch hinsichtlich der Kooperation mit der Universität eine Besonderheit darstellen. So liegen zwar klare formale Vorgaben für die Kooperation zwischen Schule und Universität vor und sind auch in den jeweiligen Ordnungen festgeschrieben; diese Vorgaben müssen aber von den Beteiligten vor Ort ausgestaltet werden. Im Rahmen der Zusammenarbeit zwischen Angehörigen der Schule und der Universität treffen auch gleichzeitig zwei Institutionen aufeinander, denen verschiedene Logiken und Ziele zugrunde liegen. Laborschule und Oberstufen-Kolleg haben dafür größtenteils identische, aber zum Teil eben auch leicht unterschiedliche Bearbeitungsmodi entwickelt und entwickeln diese stetig weiter. Resümieren lässt sich, dass die dauerhafte Kooperation einen Gewinn für die Praxisforschung und ihre Etablierung darstellt und sowohl für die Schulentwicklung als auch für den wissenschaftlichen Diskurs einen Mehrwert bereithält. 


\section{Literatur und Internetquellen}

Altrichter, H., \& Feindt, A. (2004). Zehn Fragen zur LehrerInnenforschung. In S. Rahm \& M. Schratz (Hrsg.), LehrerInnenforschung. Theorie braucht Praxis. Braucht Praxis Theorie? (S. 84-101). Innsbruck: StudienVerlag.

Altrichter, H., Posch, P., \& Spann, H. (2018). Lehrerinnen und Lehrer erforschen ihren Unterricht (5., grundlegend überarbeitete Aufl.). Bad Heilbrunn: Klinkhardt.

Bathe, S., Bernard, U., Boller, S., Kemper, A., Lau, R., \& Wäcken, M. (2010). Innere Differenzierung in der Sekundarstufe II. Abschlussbericht der Forschungs- und Entwicklungsgruppe. Bielefeld: Unveröffentlichtes Manuskript.

Boller, S., \& Lau, R. (Hrsg.). (2010). Innere Differenzierung in der Sekundarstufe II. Ein Praxishandbuch für Lehrer/innen. Weinheim: Beltz.

Bosse, D., \& Kempf, J. (2013). Der Übergang in die Einführungsphase als Herausforderung. In J. Asdonk, S. U. Kuhnen \& P. Bornkessel (Hrsg.), Von der Schule zur Hochschule: Analysen, Konzeptionen und Gestaltungsperspektiven des Übergangs (S. 89-99). Münster et al.: Waxmann.

Gass, S. M., \& Mackey, A. (2000). Stimulated Recall Methodology in Second Language Research. Mahwah, NJ: Lawrence Erlbaum Associates Publishers.

Gemeinsame Leitung des Oberstufen-Kollegs (2017). Modell für die Forschung und Entwicklung am Oberstufen-Kolleg. Beschlossen durch die Gemeinsame Leitung am 05. Juli 2017. Bielefeld: Unveröffentlichtes Manuskript.

Hahn, S., Klewin, G., Koch, B., Kuhnen, S., Palowski, M., \& Stiller, C. (im Druck). Über Praxisforschung zum Transfer von Innovationen? In P. Dobbelstein, M. Heinrich, C. Schreiner, U. Steffens, C. Wiesner, \& S. Angerer (Hrsg.), Praxistransfer in der Schulund Unterrichtsforschung. Münster et al.: Waxmann.

Hahn, S., \& Oelkers, J. (Hrsg.). (2012). Forschung und Entwicklung am Oberstufen-Kolleg. Selbst- und Peerbericht über die Entwicklungen der Versuchsschule und wissenschaftlichen Einrichtung Oberstufen-Kolleg in den Jahren 2005 bis 2010. Bad Heilbrunn: Klinkhardt.

Huber, L. (2004). LehrerInnenforschung an einer Versuchsschule - oder: Die Mühen der Ebene am Teutoburger Wald. In S. Rahm \& M. Schratz (Hrsg.), LehrerInnenforschung. Theorie braucht Praxis. Braucht Praxis Theorie? (S. 35-57). Innsbruck: StudienVerlag.

Jung-Paarmann, H. (2014a). Reformpädagogik in der Praxis. Geschichte des Bielefelder Oberstufen-Kollegs. 1969-2005. Bad Heilbrunn: Klinkhardt

Jung-Paarmann, H. (2014b). Forschung am Oberstufen-Kolleg (1970-2002). In S. Hahn, M. Heinrich \& G. Klewin (Hrsg.), Forschung und Entwicklung am Oberstufen-Kolleg. Rückblick - Bestandsaufnahme - Ausblick (S. 13-80). Münster: MV.

Keuffer, J., \& Klewin, G. (2009). Das Lehrer-Forscher-Modell am Oberstufen-Kolleg: Ausgangskonzept und heutige Praxis. In N. Hollenbach \& K.-J. Tillmann (Hrsg.), Die Schule forschend verändern. Praxisforschung aus nationaler und internationaler Perspektive (S. 203-212). Bad Heilbrunn: Klinkhardt.

Klewin, G., \& Palowski, M. (2018). (Wie) Wirkt Schulforschung auf Unterricht? Chancen und Grenzen von Praxisforschung für Unterrichts- und Schulentwicklung. Zwei Workshops im Rahmen der 2. Innsbrucker Gespräche zur Schulentwicklung, 18.-20.04.2018, Innsbruck. Unveröffentlichtes Manuskript.

Klewin, G., Schumacher, C., \& Textor, A. (2016). Der Beitrag der Praxisforschung zur Curriculumentwicklung - Erfahrungen aus Laborschule und Oberstufen-Kolleg. In M. Hallitzky, B. Koch-Priewe, A. Rakhkochkine, J.-C. Störtländer \& M. Trautmann (Hrsg.), Vergleichende Didaktik und Curriculumforschung/Comparative Research into Didactics and Curriculum (S. 262-273). Bad Heilbrunn: Klinkhardt. 
Laborschule Bielefeld (2012). Grundlagenerlass für die Aufgaben und die Zusammenarbeit von Versuchsschule, Wissenschaftlicher Einrichtung, Gemeinsamer Leitung und Wissenschaftlichem Beirat. Gemeinsamer Runderlass des Kultusministeriums und des Ministeriums für Wissenschaft und Forschung vom 13.07.1992, Stand 01.07.2012. Bielefeld: Oberstufen-Kolleg.

Lau, R., \& Große-Klußmann, D. (2012). Entwicklung und Transfer von Fortbildungskonzepten zur Inneren Differenzierung für die Sekundarstufe II. Abschlussbericht der Forschungsund Entwicklungsgruppe. Bielefeld: Unveröffentlichtes Manuskript.

Meyer, H. (in Zusammenarbeit mit W. Fichten) (2010). Gemeinsam forschen lernen. Eröffnungsvortrag der XV. Fachtagung des Nordverbunds Schulbegleitforschung, 16.09.2010, Hamburg. Unveröffentlichtes Manuskript.

MSW NRW (Ministerium für Schule und Weiterbildung Nordrhein-Westfalen) (2007). Grundordnung des Oberstufen-Kollegs des Landes Nordrhein-Westfalen an der Universität Bielefeld. Runderlass des Ministeriums für Schule und Weiterbildung vom 28. Juni 2007. Bielefeld: Oberstufen-Kolleg.

Oberstufen-Kolleg Bielefeld (2007). Verwaltungs- und Benutzungsordnung für die Wissenschaftliche Einrichtung Oberstufen-Kolleg der Fakultät für Pädagogik der Universität Bielefeld vom 1. August 2007. Bielefeld: Oberstufen-Kolleg.

Thurn, S., \& Tillmann, K.-J. (2011). Laborschule - Schule der Zukunft. In S. Thurn \& K.J. Tillmann (Hrsg.), Laborschule - Schule der Zukunft. IMPULS Laborschule, 5, 8-16.

Tillmann, K.-J. (2011). Forschung in der Versuchsschule. In S. Thurn \& K.-J. Tillmann (Hrsg.), Laborschule - Schule der Zukunft. IMPULS Laborschule, 5, 94-107.

Walther, A. (2014). Übergänge im Lebenslauf, zwischen Standardisierung und Entstandardisierung. In C. Hof, M. Meuth \& A. Walther (Hrsg.), Pädagogik der Übergänge: Übergänge in Lebenslauf und Biografie als Anlässe und Bezugspunkte von Erziehung, Bildung und Hilfe (S. 14-36). Weinheim \& Basel: Beltz Juventa.

Monika Palowski, Dr. phil., geb. 1984, wissenschaftliche Mitarbeiterin an der Wissenschaftlichen Einrichtung Oberstufen-Kolleg der Fakultät für Erziehungswissenschaft der Universität Bielefeld.

E-Mail: monika.palowski@uni-bielefeld.de

Gabriele Klewin, Dr., geb. 1962, stellvertretende Leiterin der Wissenschaftlichen Einrichtung Oberstufen-Kolleg an der Fakultät für Erziehungswissenschaft der Universität Bielefeld.

E-Mail: gabriele.klewin@uni-bielefeld.de

Anschrift: Wissenschaftliche Einrichtung Oberstufen-Kolleg, Universitätsstraße 23, 33615 Bielefeld

Johanna Gold, Dr. phil., geb. 1978, Akademische Rätin a.Z. an der Wissenschaftlichen Einrichtung Laborschule an der Fakultät für Erziehungswissenschaft der Universität Bielefeld.

E-Mail: johanna.gold@uni-bielefeld.de

Anschrift: Wissenschaftliche Einrichtung Laborschule, Universitätsstraße 21, 33615 Bielefeld 